\title{
Performance of Brain-Computer Interfacing Based on Tactile Selective Sensation and Motor Imagery
}

\author{
Lin Yao, Xinjun Sheng, Natalie Mrachacz-Kersting, \\ Xiangyang Zhu, Dario Farina, Ning Jiang*
}

\begin{abstract}
A large proportion of users do not achieve adequate control using current non-invasive Brain-computer Interfaces (BCI). This issue has being coined "BCI-Illiteracy", and is observed among BCI modalities. Here, we compare the performance and BCI-illiteracy rate of tactile selective sensation (SS) and motor imagery (MI) BCI, for large subject samples. We analyzed 80 experimental sessions from 57 subjects with two-class SS protocols. For SS, the group average performance was $79.8 \pm 10.6 \%$, with 43 out of the 57 subjects $(75.4 \%)$ exceeding the 70\% BCI-illiteracy threshold for left and right hand SS discrimination. When compared to previous results, this tactile BCI outperformed all other tactile BCIs currently available. We also analyzed 63 experiment sessions from 43 subjects with two-class MI BCI protocols, where the group average performance was $77.2 \pm 13.3 \%$, with $69.7 \%$ of the subjects exceeded the $70 \%$ performance threshold for left and right hand MI. For within-subject comparison, the 24 subjects who participated to both the SS and MI experiments, the BCI performance was superior with SS than MI especially in beta frequency band $(p<0.05)$, with enhanced $R^{2}$ discriminative information in the somatosensory cortex for the SS modality. Both SS and MI showed a functional dissociation between lower alpha ([8 10] Hz) and upper alpha ([10 13] Hz) bands, with BCI performance significantly better in the upper alpha than the lower alpha $(p<0.05)$ band. In summary, we demonstrated that SS is a promising BCI modality with low BCI illiteracy issue, and has great potential in practical applications reaching large population.
\end{abstract}

\section{INTRODUCTION}

Brain-computer Interface (BCI) provides a non-muscular communication and control channel between the brain and the external environment, which would be particularly useful for locked-in patients [1]. Without requirement of external stimulus, sensory-motor rhythms (SMR) are generated by mentally performing motor imagery (MI) of a limb [2]-[5]. The MI induced event-related desynchronization (ERD)/ synchronization (ERS) brain signals [6], [7] enable direct BCI control. With the advantage of subjective motor intention decoding, this independent BCI modality has attracted extensive interest [8]-[11]. In addition, it has been shown that brain response evoked (transient and steady-state potential) or induced (oscillatory power) by exogenous stimuli can facilitate voluntary attention decoding, resulting in at least three BCI

Lin Yao, and Ning Jiang are with Department of Systems Design Engineering, Faculty of Engineering, University of Waterloo, Waterloo, Canada (E-mail: lin.yao@uwaterloo.ca, ning.jiang@uwaterloo.ca).

Xinjun Sheng and Xiangyang Zhu are with State Key Lab of Mechanical System and Vibration, Shanghai Jiao Tong University, Shanghai, China. (xjsheng@sjtu.edu.cn, mexyzhu@sjtu.edu.cn).

Natalie Mrachacz-Kersting is with Center for Sensory-Motor Interaction, the Faculty of Medicine, Aalborg University, Aalborg, Denmark. (nm@hst.aau.dk)

Dario Farina is with Department of Bioengineering, Imperial College London, London, UK. (d.farina@imperial.ac.uk) categories: (A) transient BCI [12]-[14], such as the visual P300-based speller; (B) steady-state BCI [15]-[17], such as the steady-state visual evoked potentials (SSVEP); and (C) oscillatory BCI [18], [19], such as the tactile BCI based on stimulus-induced oscillatory dynamics. The diversity of BCI modalities may offer alternative methods when a selected modality fails in achieving an adequate performance.

As the number of experimental studies increased, it became evident that a portion of users cannot control specific BCI systems, even after extensive training [20]-[29], which has been termed "BCI Illiteracy" [26], [27]. Three major MI studies on 193 subjects [26], 80 subjects [30] and 52 subjects [31], have specifically investigated this problem. These studies reported that between $\sim 40 \%$ and $\sim 60 \%$ of the subjects investigated could achieve an average accuracy in BCI control of only $~ 50 \%$ in the classic two-class scenario [32]. A threshold in accuracy of 70\% was proposed for defining BCI-illiteracy [32]. According to this definition, visual P300 and steady-state visual evoked potential (SSVEP) based BCI systems showed substantially lower rates of BCI-illiteracy, with less training [27], [28]. However, these fast visual BCIs require the full engagement of the users' gaze control, which can be challenging and undesirable in real-life application settings. A tactile BCI provides an alternative approach to increasing current BCI diversity by fully exploring the functioning somatosensory system of the BCI user. The first prototype of a tactile BCI was proposed by Mueller-Putz et al. [33], and based on steady-state somatosensory evoked potentials (SSSEP) [34]-[36]. This tactile BCI system does not require eye control and is therefore independent of visual stimuli. SSSEP is a steady-state component of brain signals, evoked by sustained repetitive vibrotactile stimulation within the frequency range $17-35 \mathrm{~Hz}$ [37]. The evoked amplitude of the SSSEP can be modulated by subjective attention [38]. Early experimental studies have shown that the classification accuracy for this BCI modality ranged from $64 \%$ to $84 \%$, with an average accuracy of $70.4 \%$ and a high BCI-illiteracy rate [38]. A subsequent study on SSSEP showed a mean classification accuracy of 58\% for 16 subjects, with 15 of the subjects resulting in accuracy less than $70 \%$ [39]. Finally, a tactile P300 system, similar to the visual P300 BCI, based on the oddball paradigm, has also been proposed [13] and achieved an accuracy of $72 \%$ in 11 subjects, when selecting between two targets.

Recently, in a series of studies, we proposed a tactile BCI based on oscillatory dynamics from the somatosensory area of the cortex, which we termed selective sensation (SS) tactile BCI [18], [19], [40], [41]. This approach is based on the observation that mental processing of afferent inflow in the human somatosensory system also induces ERD/ERS [42], [43] [44]. We demonstrated that the integration of stimulus-induced oscillatory dynamics in tactile selective 
sensation has substantially outperformed previous tactile BCI systems [40], making tactile BCI potentially applicable to a large number of users.

BCIs using MI and SS are both based on the dynamics of brain oscillation quantified as ERD/ERS [45], [46], which are modulated during imagined movement [2], [47], [48], and during perceiving sensory stimulation [42], [43], [49], [50]. For instance, motor imagery of hand movements accompanies contralateral ERD and ipsilateral ERS [2], [51], while selective attention modulates somatosensory oscillations in the alpha and beta bands [40], [52], which results in significantly increased beta ERD/ERS due to attentional effects. These two BCIs are based on different signal modalities, with one mainly from the motor cortex (MI) and the other from the somatosensory cortex (SS). Due to the physical proximity of the motor and sensory cortex and limited selectivity of EEG, the performance of MI and SS are similar, although an hybrid of MI and SS has shown superior performance than the individual modalities [19]. However, SS has been so far tested in small subject groups. Here, we provide a systematic analysis of the SS tactile BCI performance for 80 experimental sessions, performed cumulatively in previous studies, as well as a comparison with MI BCI from 63 experimental sessions.

\section{Methodology}

\section{A. Subjects}

57 healthy, BCI-naïve subjects participated in the tactile selective sensation experiments (20 female, all right handed, average age $23.2 \pm 2.9$ years), for a total of 80 experimental sessions. 43 healthy, BCI-naïve subjects participated in the motor imagery experiments (10 female, all right handed, average age $22.2 \pm 2.5$ years), for a total of 63 experimental sessions.

The measures were approved by the Ethics Committee of the Shanghai Jiao Tong University, Shanghai, China, for SS and MI experiments) and the Ethics Committee of the University of Waterloo, Waterloo, Canada for SS experiments (ORE\#: 21997). All participants signed an informed consent form before participation.

\section{B. $E E G$}

In six out of seven experimental sets [18], [19], [23], [41],
[53]-[55] (as seen in table 1), EEG signals were recorded using a SynAmps2 system (Neuroscan, U.S.A.). A 64-channel quick-cap was used to collect 62-channel EEG signals, and the electrodes were placed according to the extended 10/20 system. The reference electrode was located on the vertex, and the ground electrode on the forehead. An analog bandwidth filter with $0.5 \mathrm{~Hz}$ to $70 \mathrm{~Hz}$ and a notch filter at $50 \mathrm{~Hz}$ were applied to the raw signals. The signals were digitally sampled at $250 \mathrm{~Hz}$.

A different EEG system was used in part of the experimental sessions. In these cases, a 32-channel wireless g.Nautilus EEG system (g.tec, Austria) was utilized. The electrodes were placed according to the extended 10/20 system. The reference electrode was located on the right earlobe, and the ground electrode on the forehead. A hardware notch filter at $60 \mathrm{~Hz}$ was applied to the raw signals. The signals were digitally sampled at $250 \mathrm{~Hz}$.

\section{Somatosensory Stimulation Methods}

Mechanical stimulation was applied to the wrists. Linear resonant actuators $(10 \mathrm{~mm}, \mathrm{C} 10-100$, Precision Microdrives Ltd., typical normalized amplitude $1.4 \mathrm{G}$ ) were used for producing vibrotactile stimulation. The stimulation device produced a $23-\mathrm{Hz}$ sine wave for the left wrist, and $27-\mathrm{Hz}$ sine wave for the right wrist. Both stimuli were modulated with a $175-\mathrm{Hz}$ sine carrier wave. These stimuli activate the Pacinian and Meissner corpuscles [56], which are sensitive to frequencies above $100 \mathrm{~Hz}$ and $20-50 \mathrm{~Hz}$, respectively. The amplitude of the vibration was individually adjusted to be between the maximum amplitude $(11.3 \mathrm{um})$ and half of the maximum amplitude at the resonant frequency. The selection of the optimal amplitude was based on individual feedback from the subject, such that they were comfortable with perceiving the vibration.

\section{Experimental Protocols for Tactile Selective Sensation}

We analyzed experimental data from four protocols as seen in Table 1. Thirty one subjects participated in the first experiment [18], which used the Neuroscan system. In these sessions, the subjects sat in a comfortable armchair in an electrical shielded room, with forearms and hands resting on the armrest. During the SS task period, sustained vibrotactile stimuli were simultaneously applied to both wrists and the subjects were

Table 1. Summation of the experiment paradigm in SS and MI. The number in the parentheses indicates the number of subjects who were also in SS

\begin{tabular}{|c|c|c|c|c|c|c|c|}
\hline & \multicolumn{4}{|c|}{ SS } & \multicolumn{3}{|c|}{ MI } \\
\hline $\begin{array}{l}\text { Experiment } \\
\text { Sets }\end{array}$ & $\begin{array}{c}\text { Experiment } \\
\text { One [18] }\end{array}$ & $\begin{array}{c}\text { Experiment } \\
\text { Two [41] }\end{array}$ & $\begin{array}{c}\text { Experiment } \\
\text { Three [53] }\end{array}$ & $\begin{array}{l}\text { Experiment } \\
\text { Four [19] }\end{array}$ & $\begin{array}{c}\text { Experiment } \\
\text { One [23], [54] }\end{array}$ & $\begin{array}{c}\text { Experiment } \\
\text { Two [55] }\end{array}$ & $\begin{array}{c}\text { Experiment } \\
\text { Three [19] }\end{array}$ \\
\hline $\begin{array}{l}\text { Paradigm } \\
\text { (Task) }\end{array}$ & SS-L, SS-R & $\begin{array}{l}\text { SS-L,SS-R, } \\
\text { SS-B, SS-S }\end{array}$ & $\begin{array}{l}\text { SS-L, SAO-L, } \\
\text { SS-R, SAO-R }\end{array}$ & $\begin{array}{l}\text { SS-L, SS-R, } \\
\text { MI-L, MI-R }\end{array}$ & MI-L, MI-R & $\begin{array}{l}\text { MIvib-L, } \\
\text { MIvib-R }\end{array}$ & $\begin{array}{l}\text { SS-L, SS-R, } \\
\text { MI-L, MI-R }\end{array}$ \\
\hline $\begin{array}{l}\text { Recording } \\
\text { Device }\end{array}$ & Neuroscan & g.Nautilus & Neuroscan & Neuroscan & Neuroscan & Neuroscan & Neuroscan \\
\hline $\begin{array}{c}\text { EEG } \\
\text { Channels }\end{array}$ & 62 & 32 & 62 & 62 & 62 & 62 & 62 \\
\hline $\begin{array}{l}\text { Subject } \\
\text { Number }\end{array}$ & 31 & 20 & 16 & 13 & $38(11)$ & 12 & $13(13)$ \\
\hline $\begin{array}{c}\text { Number of } \\
\text { Runs }\end{array}$ & 4 & 6 & 3 & 10 & 4 & 4 & 10 \\
\hline $\begin{array}{l}\text { Trials in } \\
\text { each task }\end{array}$ & 80 & 60 & 60 & 100 & 80 & 80 & 100 \\
\hline $\begin{array}{l}\text { Baseline } \\
\text { Interval for } \\
\text { ER/ERS }\end{array}$ & {$\left[\begin{array}{ll}-1 & -0.2\end{array}\right] \mathrm{s}$} & {$[-2-1.2] \mathrm{s}$} & {$[-2-1.2] \mathrm{s}$} & {$\left[\begin{array}{ll}-1 & -0.2\end{array}\right] \mathrm{s}$} & {$\left[\begin{array}{ll}-1 & -0.2\end{array}\right] \mathrm{s}$} & {$\left[\begin{array}{ll}-1 & -0.2\end{array}\right] \mathrm{s}$} & {$\left[\begin{array}{ll}-1 & -0.2\end{array}\right] \mathrm{s}$} \\
\hline
\end{tabular}


required to focus the sensation on one of the sides, following a cue. Four runs were performed, with 40 trials in each run $(20$ left-side and 20 right-side trials randomized in each run). At the beginning of each trial, a fixation cross appeared in the center of the screen. At the 1st second, a vibration burst of $200 \mathrm{~ms}$ stimulated both hands to prepare the subject for the subsequent task. At the 3rd second, a red cue arrow pointing either left or right was presented, with the left arrow corresponding to the SS-L task and right arrow corresponding to the SS-R task. This cue was superimposed on the fixation symbol and lasted for 1.5 s. The subjects were asked to perform a tactile selective sensation task following the appearance of the cue. The mental task continued until the 8th second, when the fixation cross disappeared. During the first run, there was no feedback whereas in the subsequent three runs a vibration feedback provided information on the on-line classification [18]. The feedback stimulus was applied according to the decoded task and lasted $500 \mathrm{~ms}$, e.g. if left hand task was recognized then left hand would be stimulated for $500 \mathrm{~ms}$, similarly if decoded type was right hand then right hand would be stimulated for $500 \mathrm{~ms}$. After the feedback, there was a relaxation time period of $1.5 \mathrm{~s}$. Finally, a random interval of 0 to $2 \mathrm{~s}$ separated the rest period from the next trial.

Twenty subjects participated in the second SS protocol [41], with the g.Tec system. During the SS task period, sustained vibrotactile stimuli were simultaneously applied to both wrists. Four SS tasks were performed according to different cues: 1. Selective sensation on the left hand (SS-L); 2. Selective sensation on the right hand (SS-R); 3. Bilateral selective sensation (SS-B); 4. Selective sensation suppressed (SS-S). The other aspect of the protocol were identical to the first experimental protocol, with the four cues instead of two cues. A total of 240 trials ( 60 trials for each task) were performed by the subjects in 6 runs, each of which consisted of 10 trials of each task in random order. There were 2-4 min breaks between two consecutive runs.

Sixteen subjects participated in the third experiment [53] using the Neuroscan system. Each subject seated on a comfortable armchair in an electrically shielded room. With both forearms and hands resting on the armrest, the subject limited as much as possible the eye blinking and the facial or arm muscular activations. Within each trial, the subject performed left or right SS tasks according to the cue (during task time, the left and right wrists were simultaneously stimulated), while maintaining the attention on the selected hand when the vibration stimuli were turned off (subjects performed imagined sensation when there were no stimulus, i.e. somatosensory attentional orientation (SAO)) [40]. A total of 120 trials (60 left and 60 right classes) were performed by the subjects in 3 runs, with 1-2 min between runs. The trials were similar as to the previous two experiments. The difference was that, for half of the mental tasks the vibrotactile stimulation was turned off, and for the other half tasks the stimulation was simultaneously applied to both wrists. During the first run, there was no feedback after the L-SAO and R-SAO tasks. During the subsequent two runs, a vibration feedback was provided to the subject after the SAO task, as in the previous two experiments. To this experiment, only the EEG signals with respect to SS were extracted for subsequent analysis.

Finally, 13 subjects participated in the fourth experiment [19] with EEG recorded using the Neuroscan system. SS and MI were both performed in this experiment. The subject's task was to perform MI or SS according to a given cue. The procedures were the similar as in the first experimental protocol, with the four cues instead of the previous two cues. A red cue pointing either up left (L-MI), up right (R-MI), lower left (L-SS) or lower right (R-SS) was presented visually on the computer monitor, and subjects performed the corresponding tasks. A total of 400 trials were performed by the subjects in 10 runs, and subjects rested between runs. 40 trials in each run, and R-MI, L-MI, R-SS, L-SS (defined below) were randomly arranged with ten trials each.

\section{E. Experimental Protocol in Motor Imagery}

Data from three experiments of MI were analyzed here. Thirty eight subjects participated in the first experiment [23], [54], with EEG recorded using the Neuroscan system. At the beginning of each trial, a fixation cross appeared in the screen. At the 1st second, a vibration burst of the same intensity stimulated both hands to attract the subject's attention mentally ready for the subsequent task, with the vibration time lasted for $200 \mathrm{~ms}$. Then at the $3 \mathrm{rd}$ second, a red cue bar pointing either left or right was presented, which superimposed on the fixation cross and lasted for $1.5 \mathrm{~s}$. The subjects should perform the left hand motor imagery (left-pointing bar) or right hand motor imagery (right-pointing bar) task after appearance of the cue bar. The mental task continued until to the $8^{\text {th }}$ second, at which time point the fixation cross disappeared. During the first run, there was no feedback after the termination of the mental task. In all the subsequent three runs, there would be vibration feedback. The feedback stimulus was applied according to the decoded task type, lasting for about $500 \mathrm{~ms}$. After the feedback, there was a relaxation time period lasting for about $1.5 \mathrm{~s}$, during which the subjects should get relaxed and could blink his or her eyes. Then a random time period of 0 to $2 \mathrm{~s}$ was inserted after the relaxation period to further avoid subject's adaptation, after that the next trial began.

In the second MI experiment [55] included 12 subjects. EEG was recorded using Neuroscan system. The experiment paradigm were the similar with experiment set one, except that at the 3.5th second, the vibration applied to both hands with the same intensity, till to the end of the motor imagery task.

In the third MI experiment [19], 13 subjects participated. EEG was recorded using the Neuroscan system. The experimental protocol was the same as in the fourth experiment of SS protocol which included both MI and SS tasks.

\section{F. ERD/ERS and time frequency decomposition}

Event related desynchronization (ERD) and event related synchronization (ERS) are defined as the percentage of power decrease (ERD) and power increase (ERS) in a defined frequency band in relation to a reference interval (usually taken at a different time interval) [46]. The frequency band alpha-beta of [8 26] $\mathrm{Hz}$ was adopted in this study for EEG filtering before the ERD/ERS calculation. The grand averaged ERD/ERS 
curves from all subjects of the same task were used to determine the activation and deactivation of the cortex areas involved in the mental tasks. Due to different timing of the 200-ms vibration burst, in the SS experiments 2 and 3, the reference interval for the ERD/ERS calculation was from $1.2 \mathrm{~s}$ to $2.0 \mathrm{~s}$ prior to the appearance of the cue; in SS experiments 1 and 4 it was from $0.2 \mathrm{~s}$ to $1.0 \mathrm{~s}$ prior to the appearance of the cue; and in the MI experiments 1, 2, and 3 it was from $0.2 \mathrm{~s}$ to $1.0 \mathrm{~s}$ prior to the appearance of the cue.

The EEG data was manually corrected for artifacts using the EEGLAB toolbox [57]. Trials contaminated with swallowing and physical movement artifacts (either in baseline or task interval) were excluded from the analysis. Time-frequency decomposition of each trial along each EEG channel was performed to construct the spatio-spectral-temporal structure according to the pre-defined mental tasks. It was calculated every $200 \mathrm{~ms}$ with a hanning tapper, convoluted with a modified sinusoid basis, in which the number of cycles linearly changed with frequency to achieve proper time and frequency resolution [58]. The $\mathrm{R}^{2}$ index (squared Pearson-correlation coefficient between feature and class label) [59], [60] was calculated based on the above spatio-spectral-temporal structures between different mental tasks, and used to locate the component of different EEG channels for the classification of the corresponding mental tasks. Moreover, the $\mathrm{R}^{2}$ index was averaged along the task time interval mentioned above, and along certain frequency bands, such as alpha $(8-13 \mathrm{~Hz})$, beta $(13-26 \mathrm{~Hz})$, or alpha-beta $(8-26 \mathrm{~Hz})$.

\section{G. Performance Evaluation}

Spatial filtering was adopted to reduce the number of channels and to enhance the feature discrimination among the investigated SS tasks. The spatial filters were determined with the Common Spatial Pattern (CSP) procedure, which has been extensively validated for BCI applications [61], [62]. The log-variance of the first and last three components produced by CSP were chosen as feature vectors, followed by linear discriminative analysis (LDA) for classification. As the most discriminative frequency bands are highly subject-dependent, the bands were selected from the following: lower alpha [8 10] $\mathrm{Hz}(\alpha-)$, upper alpha $\left[\begin{array}{ll}10 & 13\end{array}\right] \mathrm{Hz}(\alpha+)$, lower beta $\left[\begin{array}{ll}13 & 20\end{array}\right] \mathrm{Hz}$ ( $\beta$-), upper beta $[2026] \mathrm{Hz}(\beta+)$, alpha [8 13] Hz $(\alpha)$, beta [13 26] $\mathrm{Hz}(\beta)$, alpha-beta $\left[\begin{array}{ll}8 & 26\end{array}\right] \mathrm{Hz}(\alpha \beta)$, and eta $\left[\begin{array}{ll}10 & 16\end{array}\right] \mathrm{Hz}(\eta)$ [53]. A fourth-order Butterworth filter was applied to the raw EEG signals before the CSP spatial filtering. A $10 \times 10$ fold cross-validation was utilized to evaluate the $\mathrm{BCI}$ performance among different frequency bands, and for selecting the optimal frequency band.

EEG signals were segmented from $1 \mathrm{~s}$ to $4 \mathrm{~s}$ after the appearance of the cue (the timing interval of the 4th to 7 th seconds from the beginning of the trial) for the analysis. 60 SS-L and 60 SS-R trials were extracted for performance analysis from the above four SS experiment sets. Similarly, 60 MI-L and 60 MI-R trials were extracted for performance analysis from the above three MI experiment sets.
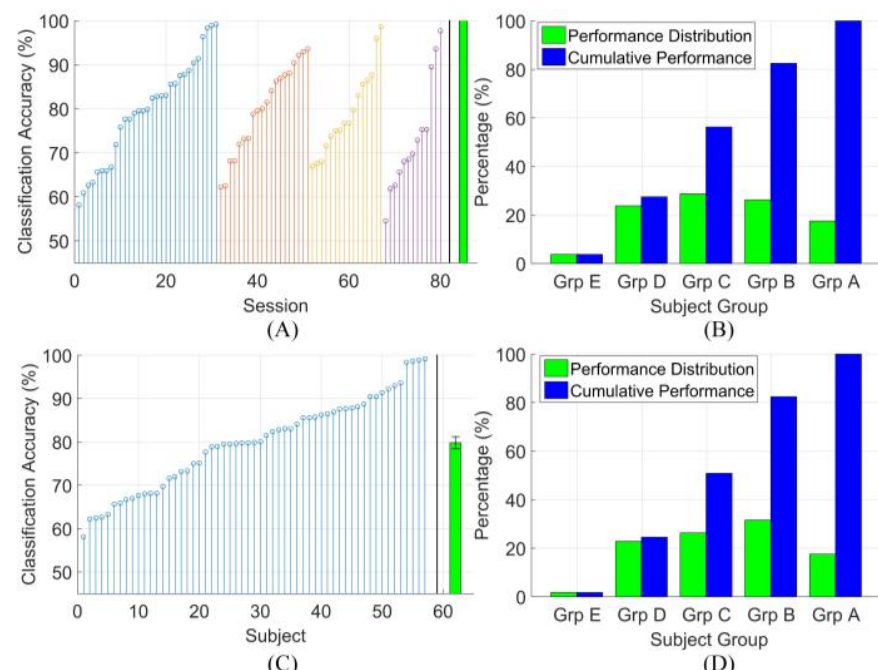

Figure 1. SS-based BCI performance. (A) BCI performance across session in different experiment sets. Each one represent one subject in one session. Green bar indicates the averaged value, the error bar indicates the standard error. Results are grouped according to experimental paradigm (light blue, orange, yellow and purple color represents experiment one, two, three and four in SS respectively), and the corresponding BCI performance is sorted with ascending order. (B) Pooled BCI performance distribution. Green bar indicates the percentage of different performance groups; blue bar indicates the cumulative distribution. Different BCI performance groups are: performance below $61.67 \%$, between $[61.67 \%$ $70 \%$ ], between [70\% 80\%], between [80\% 90\%], and above 90\%. (C) BCI performance distribution of subjects in different BCI performance groups (for those subjects participated several sessions, one of the sessions with highest performance was selected). (D) BCI performance distribution of subjects in different BCI performance groups.

\section{H. Statistics}

One-way ANOVA with repeated-measures was used to analyze differences in performance among BCI task pairs (with $p=0.05$ ), and multiple comparisons with Bonferroni correction was used for post-hoc comparison whenever the main effect was found to be significant. For the interpretation of the classification result, the theoretical chance level was corrected with the number of trials [63]. The corrected chance accuracy

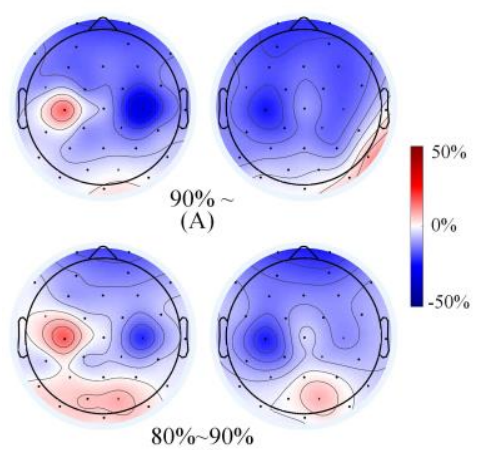

(B)

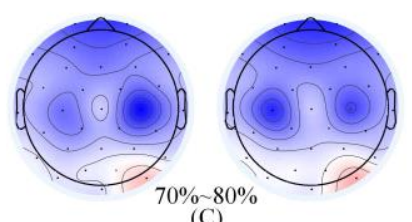

(C)
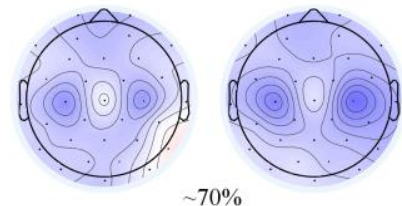

(D)
Figure 2. Grand-averaged ERD/ERS distribution within alpha-beta frequency band [8 26] Hz across different BCI performance groups, with left topography corresponds to SS-L, right topography corresponds to SS-R. (A) ERD/ERS activation in BCI group of performance above $90 \%$. (B) ERD/ERS activation in BCI group of performance between [80\% 90\%]. (C) ERD/ERS activation in BCI group of performance between [70\% 80\%]. (B) ERD/ERS activation in BCI group of performance below 70\%. Color bar indicates the ERD/ERS value. Note: ERD/ERS value is averaged between 1 to 4 second after the appearance of the cue. 
for $\mathrm{p}=0.01$ for two-class classification was $61.67 \%$. According to the BCI performance, subjects were grouped into five groups, with performance $>90 \%$ as Group $\mathrm{A}$, in the range $[80 \%$ 90\%] as Group B, [70\% 80\%] as Group C, [61.67\% 70\%] as Group D, and $<61.67 \%$ as Group E.

\section{RESULTS}

\section{A. Tactile Selective Sensation BCI performance}

Fig. 1 (A) illustrates the SS-based BCI performance of 80 sessions, grouped and color-coded by the four experiment protocols. The average classification accuracy was $78.7 \pm 11.2 \%$. Fig. 1 (B) illustrates the pooled distribution of the data shown in Fig. 1(A), with $3.75 \%$ in Group E, $23.75 \%$ in Group D, 28.75\% in Group C, 26.26\% in Group B, and 17.5 in Group A. Fig. 1 (C) illustrates the SS-based BCI performance of the 57 subjects (for those subjects participated in several sessions, the session with the highest performance was used). The average classification accuracy was $79.8 \pm 10.6 \%$. Fig. 1 (D) illustrates the pooled distribution of the data shown in Fig. 1 C), with $1.75 \%$ in Group E, $22.81 \%$ in Group D, $26.32 \%$ Group C, 31.58\% in Group B, and 17.54\% in Group A.

\section{B. Cortical Activation among Different Group in SS}

Fig. 2 illustrates the ERD/ERS distribution of SS-L and SS-R tasks across groups with different $\mathrm{BCI}$ performance. It can be seen that contralateral activation was stronger as compared to the ipsilateral activation, i.e. during the left selective sensation task the ERD of the contralateral right hemisphere (channel C4) was congruently stronger than that of the ipsilateral left hemisphere (channel C3), and vice versa for the right selective sensation. Group A (performance >90\%) has shown spatially diverse activation patterns with respect to different tasks, the ERS induced by SS-L and ERD induced by SS-R in C3 EEG channel presented a stronger discriminative brain signals for EEG classification. Similarly, Group B also exhibited similar phenomena, but with reduced ERD in C4 channel as induced by SS-L task when compared with group one. In contrast, Group C exhibited both ERD in left and right hemisphere when performing SS-L and SS-R tasks, but with stronger ERD in contralateral hemisphere than that in ipsilateral side. In the Group D, the left and right somatosensory cortexes were both activated as shown ERD in both hemisphere, but spatial

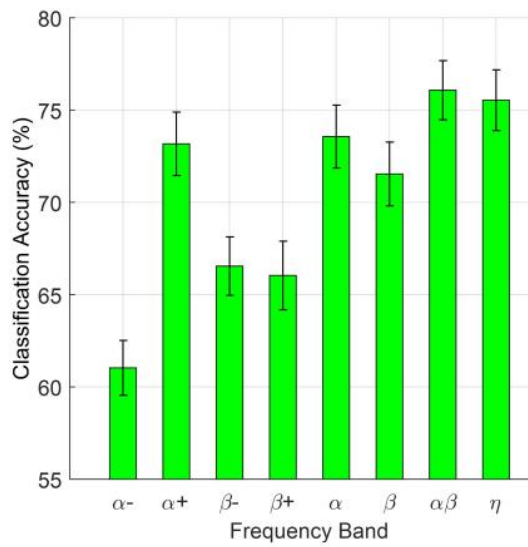

(A)

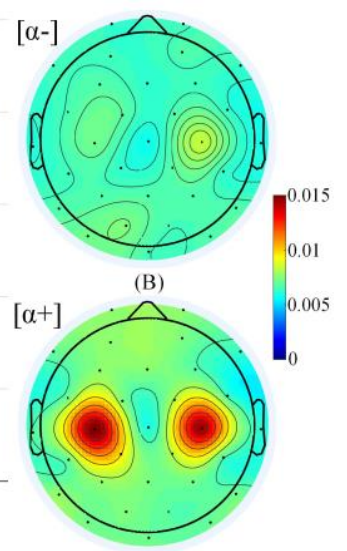

(C)
Figure 3. BCI performance in different frequency band. (A) Averaged BCI performance distribution across different frequency band (across all 57 subjects). Error bar indicates standard error. (B) Grand-averaged $\mathbf{R}^{2}$ value distribution within lower alpha frequency band. (C) Grand-averaged $R^{2}$ value distribution within upper alpha frequency band. The $R^{2}$ value was averaged between 1 to 4 second from the appearance of ${ }^{1534-4}$ the cue. Color bar indicates the $\mathbf{R}^{2}$ value.
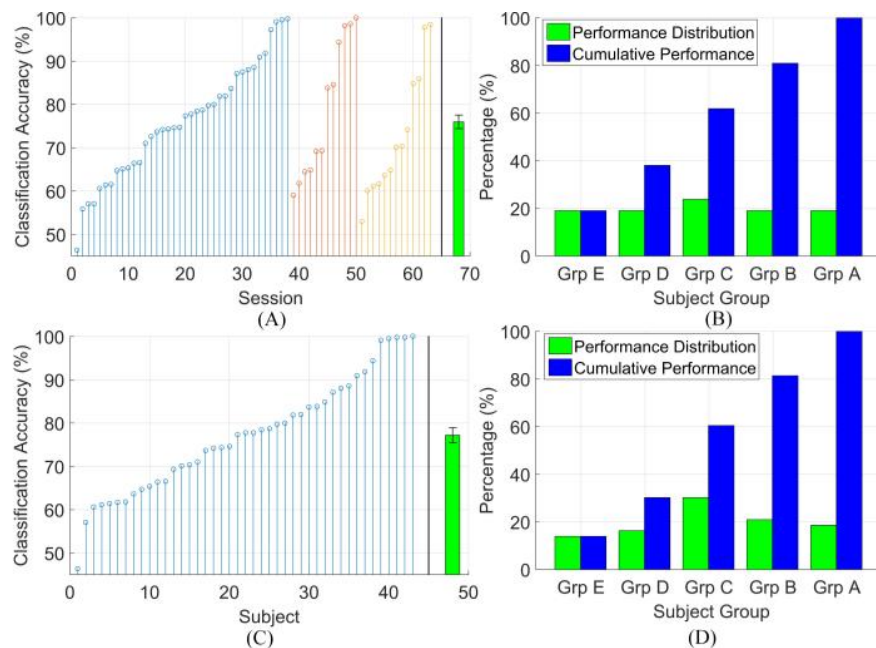

Figure 4. MI-based BCI performance. (A) BCI performance across session in different experiment sets. Each one represent one subject in one session. Green bar indicates the averaged value, the error bar indicates the standard error. Subjects are grouped according to experiment paradigm (light blue, orange and yellow color represents experiment one, two and three in MI respectively), and the corresponding BCI performance is sorted with increasing order. (B) BCI performance distribution of session in different BCI performance group. Green bar indicates the percentage of different performance group; blue bar indicates the cumulative distribution. Different BCI performance group are: performance below $61.67 \%$, between $[61.67 \% 70 \%]$, between $[70 \% 80 \%]$, between $[80 \%$ $90 \%$ ], and above $90 \%$. (C) BCI performance distribution of subjects in different BCI performance group (for those subjects participated several sessions, one of the sessions with highest performance was selected). (D) BCI performance distribution of subjects in different BCI performance group.

discrimination between tasks were less discriminative, resulting the relatively poor BCI performance.

\section{BCI Performance within Different Frequency Bands in SS}

Fig. 3 (A) illustrates the BCI performance within different frequency bands. One way ANOVA with repeated measure has shown that there was significant difference among the different frequency bands. Post-hoc testing showed that BCI
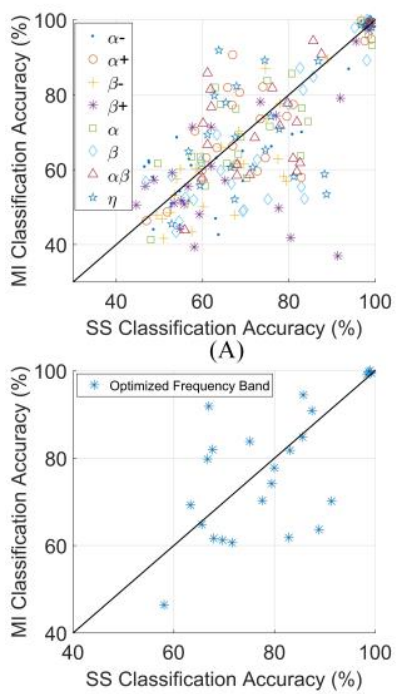

(C)

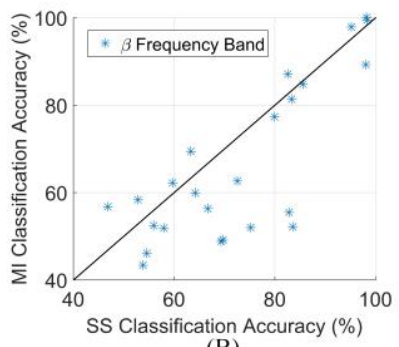

(B)

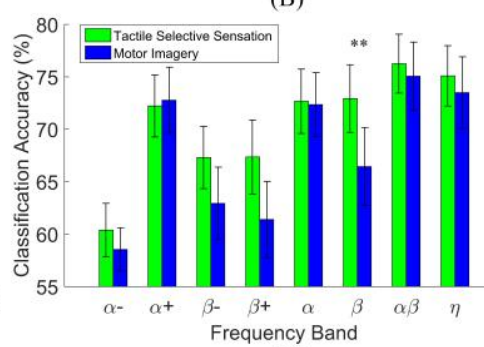

(D)

Figure 5. Comparison between SS and MI with common subjects who participated both experiments. (A) BCI performance between SS and MI among different frequency band. (B) BCI performance between SS and MI in $\beta$ frequency band. (C) BCI performance between SS and MI when subject specific frequency band was selected. (D) BCI performance in different frequency band with respect to SS and MI. Green bar represents

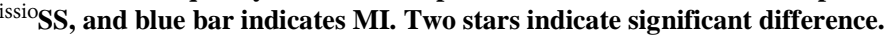


performance of upper alpha band was significant higher than that in lower alpha band. Moreover, Fig. 3 (B) (C) illustrates the $\mathrm{R}^{2}$ discriminative information distribution across the scalp, which clearly demonstrates the importance of the upper alpha band in the discrimination of SS-L and SS-R. Similarly, this also holds for the MI discrimination.

\section{BCI performance of Motor Imagery}

Fig. 4 (A) illustrates the MI-based BCI performance of 63 subjects/sessions, grouped and color-coded for the three experimental protocols. The average classification accuracy was $76.0 \pm 13.2 \%$. Fig. 4 (B) illustrates the pooled distribution of the data shown in Fig. 4 (A), with 19.05\% in Group E, 19.05\% in Group D, 23.81\% in Group C, $19.05 \%$ in Group B, and $19.05 \%$ in Group A. Fig. 4 (C) illustrates the MI-based BCI performance of 43 subjects (for those subjects that participated in several sessions, the session with the highest performance was selected). The average classification accuracy was $77.2 \pm 13.3 \%$. Fig. 4 (D) illustrates the pooled distribution of the data shown in Fig. 4(C), with $13.95 \%$ in Group E, $16.28 \%$ in Group D, 30.23\% in Group C, $20.93 \%$ in Group B, and $18.60 \%$ in Group A.
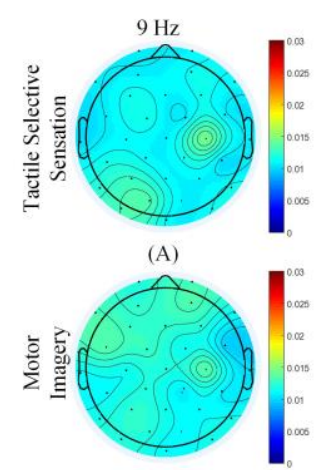

(D)

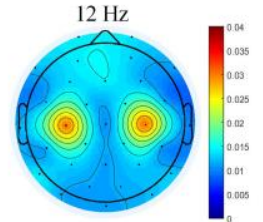

(B)

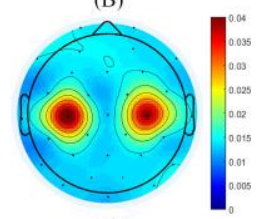

(E)

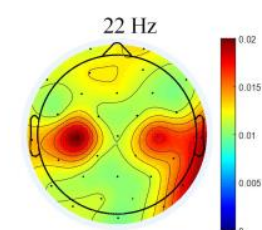

(C)

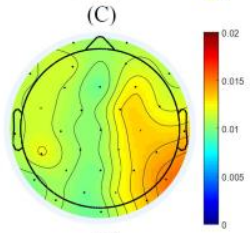

(F)
Figure 6. Grand-averaged $R^{2}$ value distribution between SS and MI within different frequency bands. (A) Grand-averaged $R^{2}$ topography with respect to SS at $9 \mathrm{~Hz}(\alpha-)$. (B) Topography with respect to SS at $12 \mathrm{~Hz}(\alpha+)$. (C) Topography with respect to $\mathrm{SS}$ at $22 \mathrm{~Hz}(\beta+)$. (D) Grand-averaged $\mathbf{R}^{2}$ topography with respect to $\mathrm{MI}$ at $9 \mathrm{~Hz}(\alpha-)$. (E) Topography with respect to MI at $12 \mathrm{~Hz}\left(\alpha^{+}\right)$. (F) Topography with respect to MI at $22 \mathrm{~Hz}(\beta+)$. The $\mathbf{R}^{2}$ value was averaged between 1 to 4 second from the appearance of the cue. Color bar indicates the $\mathbf{R}^{2}$ value.

\section{E. Subjects in both SS and MI Data}

Fig. 5 is the analysis of the comparison of the performance between SS and MI among subjects who participated in both experiments $(\mathrm{n}=24)$. Fig. 5 (A) illustrates the performance correlation between SS and MI within different frequency band. It has shown that there was a linear correlation between them with R-square $=0.54(p<0.05)$, and paired- $t$ test has shown that SS was significantly higher than MI $(p=0.002)$. Fig 5 (B) shows the correlation of SS and MI in beta frequency band only, and in this frequency band the SS performance was significantly higher than MI $(\mathrm{P}<0.05)$. Fig 5 (C) shows the SS and MI when subject specific frequency band was selected, and no significant difference in performance was found (paired- $t$ test, $p=0.49$ ). In this scenario, 17 subjects $\geq 70 \%$ in SS, 16 subjects $\geq 70 \%$ in MI, and 20 subjects $\geq 70 \%$ when either SS or
MI was selected. Moreover, Fig 5 (D) illustrates that the lower alpha $\left[\begin{array}{ll}8 & 10\end{array}\right] \mathrm{Hz}(\alpha-)$ and upper alpha $\left[\begin{array}{lll}10 & 13\end{array}\right] \mathrm{Hz}\left(\alpha^{+}\right)$frequency bands showed a significance BCI performance dissociation both in SS and MI $(\mathrm{P}<0.05)$. There was a significance difference in BCI performance between SS and MI when the beta $\left[\begin{array}{ll}13 & 26\end{array}\right] \mathrm{Hz}(\beta)$ frequency band was utilized for BCI performance evaluation $(\mathrm{P}<0.05)$, which was also shown in Fig. 5 (B). Fig. 6 shows the grand averaged discriminative $\mathrm{R}^{2}$ value distribution among different frequency bands. The $12 \mathrm{~Hz}$ frequency showed similar spatial distribution between SS and MI within the left and right sensorimotor cortex. In contrast, the $22 \mathrm{~Hz}$ frequency component showed a spatial distribution within the left and right somatosensory cortex specific for SS.

\section{DISCUSSION}

\section{A. BCI-Illiteracy problem in Tactile BCI}

A group of 53 subjects with 80 session SS experiments revealed that the tactile BCI can achieve an averaged accuracy of $79.8 \pm 10.6 \%$, with $27.5 \%$ of the investigated population below the $70 \%$ critical level of accuracy. Without the requirement of eye movement or focus control, tactile BCI opened new avenue for BCI development, which will be especially useful to those locked-in patients but with partly preserved somatosensory functionality. In the existed tactile BCI systems in the literature, the tactile stimulus evoked transient [13] and steady-state potential [33], [64] were utilized for BCI construction, reaching a mean BCI performance of $72 \%, 70.4 \%$ and $58 \%$ respectively, with much higher BCI-illiteracy rate of $90 \%$ and $93.75 \%$. In contrast, in our proposed tactile selective sensation $\mathrm{BCI}$, the stimulus-induced oscillatory dynamics was utilized for BCI construction, which has demonstrated a significantly enhanced tactile BCI performance when compared with existing tactile BCI modalities in the literature [13], [33], [64], [65], with much higher classification accuracy in a much larger experiment subject group and much lower BCI-illiteracy rate.

Combining the tactile evoked SSSEP and transient ERP brain responses, a hybrid three-class tactile BCI was recently proposed by Breitwieser et al. [66], with an average accuracy of $57 \%$, showing an improved BCI performance when comparing with solely SSSEP or ERP. The SSSEP response reflects the somatic information processing. It has a frequency specific feature, which is related to the stimulation frequency [34], [38]. In contrast, the ERD/ERS oscillatory dynamics reflects somatosensory processing, and has a non-stimulation frequency specific feature [50]. Therefore, the ERD/ERS oscillatory response and SSSEP response provide complementary information of the somatosensory input processing. Thus theoretically, in tactile BCIs, hybridizing the oscillatory dynamics and SSSEP response would provide a way to improve current tactile BCI performance. This would be worthy of future investigation.

In the investigated 57 subject SS BCI group, the cortical activation map has shown different oscillatory dynamics among subject groups with different $\mathrm{BCI}$ performance ranges, i.e. BCI group with performance above $90 \%$ (Group One), within [80\% 90\%] (Group Two), [70\%-80\%] (Group Three), and below 70\% (Group Four) as shown in Fig. 2. Contralateral somatosensory activation (ERD) was present in all four BCI 
groups. During the SS-L task, the ERD of the right hemisphere (C4) was stronger than that of the left hemisphere (C3), and vice versa for the SS-R task. Both group one and group two have shown a more pronounced ERS on the left hemisphere as compared to group three and group four when performing SS-L task. The SS-L induced ERD in contralateral right hemisphere was stronger in group one than that in group two. Moreover, group two exhibited a stronger ERS in occipital region than that in group one. The contrasted activation pattern during SS-L and SS-R were clear in spatial distribution among group one, group two and group three, which showed a BCI decoding performance above $70 \%$, while to those considered as BCI-illiteracy subjects the spatial contrast between SS-L and SS-R was lower than the subjects with performance above $70 \%$. In this study, sustained tactile stimulation were applied to subjects' wrists, different stimulation pattern and body parts would provide some new way to further improve tactile performance, especially those considered as BCI-illiteracy subjects.

\section{B. Comparison with oscillatory MI BCI}

There were sustained research efforts to further improve MI performance and reduce the number of BCI deficiency users. Machine learning algorithm on MI detection has been largely improved through several BCI competitions, and the Common Spatial Pattern (CSP) is now the most recognized one in MI detection [9], [10]. However, recent studies have reported gains in accuracy of approximately 5\% when using CSP extensions and optimized spatial-spectrum filtering based on mutual information [11]. Nevertheless some users still fail to reach the acceptable level of accuracy, which is often set to $70 \%$, even with state-of-the art algorithms [67], [68]. In addition to machine learning algorithms that can better extract subject-specific pattern, subject training in modulating rhythmic activity [69], and coadaptation of the subject and algorithms [20] have all been shown to reduce the number of the poorly performing BCI users to some extent. In our accumulated MI experiments, the MI illiteracy rate was 30.3\%, which was comparable to results reported in the literature [32]. In the offline performance evaluation, subject's specific frequency band was optimally selected which reduced the MI illiterate rate to $30.3 \%$. In our previous study about the MI and MI with vibration, the mechanical stimulation effect on MI was evaluated, and in group-level no significant difference in performance was found between MI with stimulation and MI without stimulation [55]. Therefore in current MI subject group, MI with stimulation in MI experiment set two and three were pooled together with MI without stimulation in experiment set one.

Contrary to MI BCI using brain signal mainly from motor cortex, in our tactile BCI, brain signal from somatosensory cortex was exploited to decode subjective sensation intention. The BCI-illiteracy rate was $24.56 \%$, which was approximately $20 \%$ lower than existed large MI group studies [32], and a $5.76 \%$ lower than MI group in current study. Only $1.57 \%$ of the subjects' SS performance was lower than $61.67 \%$, which was considered as random guess, in a two-class scenario. This number is much lower than the corresponding portion: $13.95 \%$. In an earlier randomized controlled study with a smaller number of subjects, we demonstrated that the SS BCI performance was comparable to MI BCI [19]. Further, in the same study, we showed that a hybrid BCI combining SS and MI had significant lower BCI-illiteracy rate that using SS or MI separately: (63.64\% in MI, 54.55\% in SS, and 9.09\% in hybrid modality (without subject specific frequency band selection).

We observed that the beta ([13 26] Hz) frequency band played different role in SS and MI classification. In the 24 subjects participated both in SS and MI, the performance of beta frequency band in SS was significantly higher than that in MI. Discriminative brain pattern analysis has shown that the $\mathrm{R}^{2}$ in SS was more pronounced around the left and right somatosensory cortex in SS than that in MI. Moreover, lower alpha band and upper alpha band has shown significant difference in performance both in MI and SS, i.e. the performance of upper alpha band was significantly higher than that in lower alpha frequency band. This observation is also reported in our earlier study on SS [18], and is consistent with previous reports in the literature[70] during MI study.

The performance correlation between SS and MI was found, which has shown a significant linear correlation with R-square $=0.54(\mathrm{r}=0.73)$, indicating the performance of SS can be a predictor for MI performance. In [30], a neurophysiological predictor of BCI performance was proposed, that is, the BCI performance could be predicted within two minutes of recording a 'relax with eyes open' condition. A correlation of $\mathrm{r}$ $=0.53$ between the predictor and the $\mathrm{BCI}$ performance was found. Moreover, our previous study also showed that a linear correlation existed between illusory stimulation and MI, with $\mathrm{R}$-square $=0.47(\mathrm{r}=0.69)$ [23]. In addition to the performance correlation between SS and MI, SS and MI provides complementary information to further increase BCI performance [19].

\section{Limitation}

In this large group study, the ages of subjects were in the range of 19 to 42 , but mainly concentrated on younger subjects, with an average of 22 years-old. Subjects were mainly male and most of them were right-handed. In future studies, subjects across different ages, and the balance of mixed gender and handedness should be considered.

In the current analysis, offline performance evaluation was performed by pooling data sets obtained from several different experimental protocols. Some of the MI data was acquired while SS was also performed (i.e. vibration stimulation activating or preconditioning sensory cortex before MI). The data was collected in different countries with different EEG systems. The variation between different experimental sets may be considered as a factor that tests the robustness of SS-based BCI performance.

By performing only imagined movement of his/her own body, MI might be applicable to a wider spectrum of patients as it does not require completely preserved sensation from the body. Any neurological condition, which affects proprioception to some degree, might affect performance of SS-based BCI. The potential and confounding factors for SS-based BCIs for patients, such as those that have suffered from a stroke, would be worthy of future investigation. 


\section{CONCLUSION}

In this work, we evaluated the "BCI-Illiteracy" problem of SS-based BCI, through a meta-analysis data consisted 80 sessions from 57 subjects. We showed that, for SS modality, the average classification accuracy in the two-class scenario was $79.8 \%$, with BCI-illiteracy rate of $24.56 \%$. Beta frequency band $\left(\left[\begin{array}{ll}13 & 26\end{array}\right] \mathrm{Hz}\right)$ showed a significantly higher BCI performance in SS than that in MI. Only 1 out of the $57(1.57 \%)$ subjects has an accuracy lower than $61.67 \%$ (random level), while MI modality, this number was 6 out of $43(13.95 \%)$. The somatosensory BCI based on stimulus-induced oscillatory dynamics provides a new signal modality for enhanced tactile BCI development.

\section{ACKNOWLEDGEMENT}

We thank all volunteers for their participation in the study. This work is supported by the University Starter Grant of the University of Waterloo (No. 203859), the National Natural Science Foundation of China (Grant No. 51620105002).

\section{REFERENCE}

[1] J. R. Wolpaw, N. Birbaumer, D. J. McFarland, G. Pfurtscheller, T. M. Vaughan, and others, "Brain-computer interfaces for communication and control," Clin. Neurophysiol., vol. 113, no. 6, pp. 767-791, 2002.

[2] G. Pfurtscheller, C. Neuper, and others, "Motor imagery activates primary sensorimotor area in humans.," Neurosci. Lett., vol. 239, no. 2-3, p. 65, 1997.

[3] G. Pfurtscheller and C. Neuper, "Motor imagery and direct brain-computer communication," Proc. IEEE, vol. 89, no. 7, pp. 1123-1134, 2001.

[4] S. N. Baker, "Oscillatory interactions between sensorimotor cortex and the periphery," Curr. Opin. Neurobiol., vol. 17, no. 6, pp. 649-655, 2007.

[5] Han Yuan and Bin He, "Brain-Computer Interfaces Using Sensorimotor Rhythms: Current State and Future Perspectives," IEEE Trans. Biomed. Eng., vol. 61, no. 5, pp. 1425-1435, 2014.

[6] G. Pfurtscheller, "Spatiotemporal ERD/ERS patterns during voluntary movement and motor imagery," Suppl. Clin. Neurophysiol., vol. 53, pp. 196-198, 2000.

[7] G. Pfurtscheller, C. Brunner, A. Schlögl, and F. H. Lopes da Silva, "Mu rhythm (de)synchronization and EEG single-trial classification of different motor imagery tasks," Neuroimage, vol. 31, no. 1, pp. 153-159, 2006.

[8] B. Blankertz, K.-R. Muller, G. Curio, T. M. Vaughan, G. Schalk, J. R. Wolpaw, A. Schlogl, C. Neuper, G. Pfurtscheller, T. Hinterberger, and others, "The BCI competition 2003: progress and perspectives in detection and discrimination of EEG single trials," IEEE Trans. Biomed. Eng., vol. 51, no. 6, pp. 1044-1051, 2004.

[9] B. Blankertz, K.-R. Muller, D. J. Krusienski, G. Schalk, J. R. Wolpaw, A. Schlogl, G. Pfurtscheller, J. R. Millan, M. Schroder, and N. Birbaumer, "The BCI competition III: Validating alternative approaches to actual BCI problems," IEEE Trans. Neural Syst. Rehabil. Eng., vol. 14, no. 2, pp. 153-159, 2006.

[10] M. Tangermann, K.-R. Müller, A. Aertsen, N. Birbaumer, C. Braun, C. Brunner, R. Leeb, C. Mehring, K. J. Miller, G. R. Müller-Putz, and others, "Review of the BCI competition IV," Front. Neurosci., vol. 6, 2012.

[11] J. Meng, L. Yao, X. Sheng, D. Zhang, and X. Zhu, "Simultaneously optimizing spatial spectral features based on mutual information for EEG classification," IEEE Trans. Biomed. Eng., vol. 62, no. 1, pp. 227-240, 2015.

[12] F. Nijboer, A. Furdea, I. Gunst, J. Mellinger, D. J. McFarland, N. Birbaumer, and A. Kübler, "An auditory brain--computer interface (BCI)," J. Neurosci. Methods, vol. 167, no. 1, pp. 43-50, 2008.

[13] A.-M. Brouwer and J. B. F. Van Erp, "A tactile P300 brain-computer interface," Front. Neurosci., vol. 4, p. 19, 2010.

[14] E. Donchin, K. M. Spencer, and R. Wijesinghe, "The mental prosthesis: assessing the speed of a P300-based brain-computer interface," Rehabil. Eng. IEEE Trans., vol. 8, no. 2, pp. 174-179, 2000.

[15] M.-A. Lopez, H. Pomares, F. Pelayo, J. Urquiza, and J. Perez, "Evidences of cognitive effects over auditory steady-state responses by means of artificial neural networks and its use in brain--computer interfaces," Neurocomputing, vol. 72, no. 16, pp. 3617-3623, 2009.

[16] M. Cheng, X. Gao, S. Gao, and D. Xu, "Design and implementation of a brain-computer interface with high transfer rates," IEEE Trans. Biomed. Eng., vol. 49, no. 10, pp. 1181-1186, 2002.

[17] G. R. Müller-putz, R. Scherer, C. Neuper, and G. Pfurtscheller, "Steady-State Somatosensory Evoked Potentials : Suitable Brain Signals for Brain - Computer Interfaces ?," vol. 14, no. 1, pp. 30-37, 2006.

[18] L. Yao, J. Meng, D. Zhang, X. Sheng, and X. Zhu, "Selective Sensation Based Brain-Computer Interface via Mechanical Vibrotactile Stimulation," PLoS One, vol. 8, no. 6, 2013.

[19] L. Yao, J. Meng, D. Zhang, X. Sheng, and X. Zhu, "Combining motor imagery with selective sensation toward a hybrid-modality BCI," IEEE Trans. Biomed. Eng., vol. 61, no. 8, pp. 2304-2312, 2014.

[20] C. Vidaurre, C. Sannelli, K.-R. Müller, and B. Blankertz,

"Co-adaptive calibration to improve BCI efficiency," J. Neural Eng., vol. 8, no. 2, p. $25009,2011$.

[21] C. Vidaurre and B. Blankertz, "Towards a cure for BCI illiteracy," Brain Topogr., vol. 23, no. 2, pp. 194-198, 2010.

[22] B. Z. Allison, C. Brunner, V. Kaiser, G. R. Müller-Putz, C. Neuper, and G. Pfurtscheller, "Toward a hybrid brain--computer interface based on imagined movement and visual attention," J. Neural Eng., vol. 7, no. 2, p. 26007, 2010.

[23] L. Yao, J. Meng, X. Sheng, D. Zhang, and X. Zhu, "A novel calibration and task guidance framework for motor imagery BCI via a tendon vibration induced sensation with kinesthesia illusion.," $J$. Neural Eng., vol. 12, no. 1, p. 16005, Dec. 2015.

[24] B. Z. Allison and C. Neuper, "Could anyone use a BCI?," Brain-computer interfaces, pp. 35-54, 2010.

[25] G. Edlinger, B. Z. Allison, and C. Guger, "How many people can use a BCI system?," in Clinical Systems Neuroscience, 2015, pp. 33-66. C. Guger, G. Edlinger, W. Harkam, I. Niedermayer, and G.

Pfurtscheller, "How many people are able to operate an EEG-based brain-computer interface (BCI)?," Neural Syst. Rehabil. Eng. IEEE Trans., vol. 11, no. 2, pp. 145-147, 2003.

[27] C. Guger, S. Daban, E. Sellers, C. Holzner, G. Krausz, R. Carabalona, F. Gramatica, and G. Edlinger, "How many people are able to control a P300-based brain--computer interface (BCI)?," Neurosci. Lett., vol. 462, no. 1, pp. 94-98, 2009.

[28] B. Allison, T. Luth, D. Valbuena, A. Teymourian, I. Volosyak, and A. Graser, "BCI Demographics: How many (and what kinds of) people can use an SSVEP BCI?," Neural Syst. Rehabil. Eng. IEEE Trans., vol. 18, no. 2, pp. 107-116, 2010.

[29] C. Guger, B. Z. Allison, B. Großwindhager, R. Prückl, C. Hintermüller, C. Kapeller, M. Bruckner, G. Krausz, and G. Edlinger, "How many people could use an SSVEP BCI?," Front. Neurosci., vol. 6, no. NOV, pp. 2-7, 2012.

[30] B. Blankertz, C. Sannelli, S. Halder, E. M. Hammer, A. Kübler, K.-R. Müller, G. Curio, and T. Dickhaus, "Neurophysiological predictor of SMR-based BCI performance," Neuroimage, vol. 51, no. 4, pp. 1303-1309, 2010.

[31] M. Ahn, H. Cho, S. Ahn, and S. C. Jun, "High theta and low alpha powers may be indicative of BCI-illiteracy in motor imagery," PLoS One, vol. 8, no. 11, 2013.

[32] M. Ahn and S. C. Jun, "Performance variation in motor imagery brain-computer interface: A brief review," J. Neurosci. Methods, vol. 243, pp. 103-110, 2015.

[33] G. R. Müller-Putz, R. Scherer, C. Neuper, and G. Pfurtscheller, "Steady-state somatosensory evoked potentials: Suitable brain signals for brain-computer interfaces?," IEEE Trans. Neural Syst. Rehabil. Eng., vol. 14, no. 1, pp. 30-37, 2006.

[34] S. Tobimatsu, Y. M. Zhang, and M. Kato, "Steady-state vibration somatosensory evoked potentials: physiological characteristics and tuning function," Clin. Neurophysiol., vol. 110, no. 11, pp. 1953-1958, Nov. 1999.

[35] M. Severens, J. Farquhar, P. Desain, J. Duysens, and C. Gielen, "Transient and steady-state responses to mechanical stimulation of different fingers reveal interactions based on lateral inhibition," Clin. Neurophysiol., vol. 121, no. 12, pp. 2090-2096, 2010. 
[36] C. Nangini, B. Ross, F. Tam, and S. J. Graham, "Magnetoencephalographic study of vibrotactile evoked transient and steady-state responses in human somatosensory cortex," Neuroimage, vol. 33, no. 1, pp. 252-262, 2006.

[37] C. Breitwieser, V. Kaiser, C. Neuper, and G. R. Müller-Putz, "Stability and distribution of steady-state somatosensory evoked potentials elicited by vibro-tactile stimulation.," Med. Biol. Eng. Comput., vol. 50, no. 4, pp. 347-57, Apr. 2012.

[38] C.-M. Giabbiconi, N. J. Trujillo-Barreto, T. Gruber, and M. M Müller, "Sustained spatial attention to vibration is mediated in primary somatosensory cortex.," Neuroimage, vol. 35, no. 1, pp. 255-62, Mar. 2007.

[39] S. Ahn, M. Ahn, H. Cho, and S. Chan Jun, "Achieving a hybrid brain-computer interface with tactile selective attention and motor imagery," J. Neural Eng., vol. 11, no. 6, p. 66004, Dec. 2014.

[40] L. Yao, X. Sheng, D. Zhang, N. Jiang, D. Farina, and X. Zhu, "A BCI System Based on Somatosensory Attentional Orientation," IEEE Trans. Neural Syst. Rehabil. Eng., vol. 25, no. 1, pp. 81-90, Jan. 2017.

[41] L. Yao, M. L. Chen, X. Sheng, N. Mrachacz-Kersting, X. Zhu, D. Farina, and N. Jiang, "A multi-class tactile brain-computer interface based on stimulus-induced oscillatory dynamics," Neural Syst. Rehabil. Eng. IEEE Trans., p. 10.1109/TNSRE.2017.2731261, 2017.

[42] N. Sharma, V. M. Pomeroy, and J.-C. Baron, "Motor imagery: a backdoor to the motor system after stroke?," Stroke., vol. 37, no. 7, pp. 1941-52, Jul. 2006.

[43] E. Houdayer, a Degardin, J. Salleron, J. L. Bourriez, L. Defebvre, F. Cassim, and P. Derambure, "Movement preparation and cortical processing of afferent inputs in cortical tremor: an event-related (de)synchronization (ERD/ERS) study.," Clin. Neurophysiol., vol. 123, no. 6, pp. 1207-15, Jun. 2012.

[44] C. Dockstader, D. Cheyne, and R. Tannock, "Cortical dynamics of selective attention to somatosensory events.," Neuroimage, 2010.

[45] G. Pfurtscheller and F. H. da Silva, "Event-related EEG/MEG synchronization and desynchronization: basic principles," Clin. Neurophysiol., vol. 110, no. 11, pp. 1842-1857, 1999.

[46] B. Graimann, J. E. Huggins, S. P. Levine, and G. Pfurtscheller, "Visualization of significant ERD/ERS patterns in multichannel EEG and ECoG data," Clin. Neurophysiol., vol. 113, no. 1, pp. 43-47, 2002.

[47] F. Cassim, W. Szurhaj, H. Sediri, D. Devos, J. L. Bourriez, I. Poirot, P. Derambure, L. Defebvre, and J. D. Guieu, "Brief and sustained movements: differences in event-related (de) synchronization (ERD/ERS) patterns," Clin. Neurophysiol., vol. 111, no. 11, pp. 2032-2039, 2000.

[48] G. Pfurtscheller, C. Neuper, C. Brunner, F. L. da Silva, and others, "Beta rebound after different types of motor imagery in man.," Neurosci. Lett., vol. 378, no. 3, p. 156, 2005.

[49] G. Pfurtscheller, M. Woertz, G. Müller, S. Wriessnegger, K. Pfurtscheller, and others, "Contrasting behavior of beta event-related synchronization and somatosensory evoked potential after median nerve stimulation during finger manipulation in man.," Neurosci. Lett., vol. 323, no. 2, p. 113, 2002.

[50] E. Houdayer, E. Labyt, F. Cassim, J. L. Bourriez, and P. Derambure, "Relationship between event-related beta synchronization and afferent inputs: Analysis of finger movement and peripheral nerve stimulations," Clin. Neurophysiol., vol. 117, no. 3, pp. 628-636, 2006.

[51] G. Pfurtscheller, C. Neuper, D. Flotzinger, and M. Pregenzer, "EEG-based discrimination between imagination of right and left hand movement," Electroencephalogr. Clin. Neurophysiol., vol. 103, no. 6, pp. 642-651, 1997.

[52] C. Dockstader, D. Cheyne, and R. Tannock, "Cortical dynamics of selective attention to somatosensory events.," Neuroimage, vol. 49, no. 2, pp. 1777-85, Jan. 2010.

[53] L. Yao, X. Sheng, D. Zhang, N. Jiang, D. Farina, and X. Zhu, "A BCI System Based on Somatosensory Attentional Orientation," IEEE
Trans. Neural Syst. Rehabil. Eng., vol. 25, no. 1, pp. 81-90, Jan. 2017.

[54] L. Yao, X. Sheng, D. Zhang, N. Jiang, N. Mrachacz-Kersting, X. Zhu, and D. Farina, "A stimulus-independent hybrid BCI based on Motor Imagery and Somatosensory Attentional Orientation," IEEE Trans. Neural Syst. Rehabil. Eng., pp. 1-1, 2017.

[55] L. Yao, X. Sheng, J. Meng, D. Zhang, and X. Zhu, "Mechanical vibrotactile stimulation effect in motor imagery based brain-computer interface," in Engineering in Medicine and Biology Society (EMBC), 2013 35th Annual International Conference of the IEEE, 2013, pp. 2772-2775.

[56] C. Breitwieser, V. Kaiser, C. Neuper, and G. R. Müller-Putz, "Stability and distribution of steady-state somatosensory evoked potentials elicited by vibro-tactile stimulation," Med. Biol. Eng. Comput., pp. 1-11, 2012.

[57] A. Delorme, T. Mullen, C. Kothe, Z. A. Acar, N. Bigdely-Shamlo, A. Vankov, and S. Makeig, "EEGLAB, SIFT, NFT, BCILAB, and ERICA: new tools for advanced EEG processing," Comput. Intell. Neurosci., vol. 2011, p. 10, 2011.

[58] R. Oostenveld, P. Fries, E. Maris, and J. M. Schoffelen, "FieldTrip: open source software for advanced analysis of MEG, EEG, and invasive electrophysiological data," Comput. Intell. Neurosci., vol. 2011, p. 1, 2011.

[59] T. H. Wonnacott and R. J. Wonnacott, Introductory statistics, vol. 19690. Wiley Chichester, 1990.

[60] G. Schalk, D. J. McFarland, T. Hinterberger, N. Birbaumer, and J. R. Wolpaw, "BCI2000: a general-purpose brain-computer interface (BCI) system," IEEE Trans. Biomed. Eng., vol. 51, no. 6, pp. 1034-1043, 2004.

[61] H. Ramoser, J. Muller-Gerking, and G. Pfurtscheller, "Optimal spatial filtering of single trial EEG during imagined hand movement," IEEE Trans. Rehabil. Eng., vol. 8, no. 4, pp. 441-446, 2000.

[62] B. Blankertz, R. Tomioka, S. Lemm, M. Kawanabe, and K.-R. Muller, "Optimizing spatial filters for robust EEG single-trial analysis," IEEE Signal Process. Mag., vol. 25, no. 1, pp. 41-56, 2008.

[63] G. R. Müller-putz, R. Scherer, C. Brunner, R. Leeb, and G. Pfurtscheller, "Better than random? A closer look on BCI results," Int. Jouranl Bioelectromagn., vol. 10, no. 1, pp. 52-55, 2008.

[64] S. Ahn, M. Ahn, H. Cho, and S. C. Jun, "Achieving a hybrid brain--computer interface with tactile selective attention and motor imagery," J. Neural Eng., vol. 11, no. 6, p. 66004, 2014.

[65] S. Ahn, K. Kim, and S. C. Jun, "Steady-State Somatosensory Evoked Potential for Brain-Computer Interface--Present and Future," Front. Hum. Neurosci., vol. 9, p. 716, 2015.

[66] C. Breitwieser, C. Pokorny, and G. R. Müller-Putz, "A hybrid three-class brain-computer interface system utilizing SSSEPs and transient ERPs," J. Neural Eng., vol. 13, no. 6, p. 66015, 2016.

[67] V. Kaiser, G. Bauernfeind, A. Kreilinger, T. Kaufmann, A. Kübler, C. Neuper, and G. R. Müller-Putz, "Cortical effects of user training in a motor imagery based brain--computer interface measured by fNIRS and EEG," Neuroimage, vol. 85, pp. 432-444, 2014.

[68] F. Pichiorri, F. D. V. Fallani, F. Cincotti, F. Babiloni, M. Molinari, S. C. Kleih, C. Neuper, A. Kübler, and D. Mattia, "Sensorimotor rhythm-based brain--computer interface training: the impact on motor cortical responsiveness," J. Neural Eng., vol. 8, no. 2, p. 25020, 2011.

[69] A. J. Doud, J. P. Lucas, M. T. Pisansky, and B. He, "Continuous three-dimensional control of a virtual helicopter using a motor imagery based brain-computer interface," PLoS One, vol. 6, no. 10, p. e26322, 2011.

[70] C. Neuper, R. Scherer, S. Wriessnegger, and G. Pfurtscheller, "Motor imagery and action observation: Modulation of sensorimotor brain rhythms during mental control of a brain-computer interface," Clin. Neurophysiol., vol. 120, no. 2, pp. 239-247, 2009. 